\title{
Improving health self-efficacy at a community health fair in Lubbock, Texas
}

\author{
Taylor H. Lindgren MBA, Chelsea Burroughs, Mark Burroughs, George Parker MBA, Fatma Levent MD
}

\begin{abstract}
To improve the health self-efficacy of the Lubbock, Texas community, Texas Tech University Health Sciences Center School of Medicine students organized a health fair to promote patient education and improve awareness of health issues, such as diabetes, heart health, hypertension, skin care, hypercholesterolemia, nutrition, stress management, cancer screening and recommendations, physical fitness, and mental health. An evaluation of 57 health fair participants' self-reported self-efficacy on a scale from 0 to 10 before and after visiting the health fair revealed an average increase in self-efficacy of 0.87 (before: 7.96 , after: 8.83, $p<0.0001, s t d=1.78$ ). Although the health fair was successful in marginally improving patient self-efficacy, patient education and overall community health can continue to be improved by expanding educational opportunities for health promotion and illness prevention, furthering overall access to care, and broadening the health fair's target population.
\end{abstract}

Keywords: self-efficacy, health, fair, prevention, education, community, rural

\section{INTRODUCTION AND BACKGROUND}

Preventive medicine is the foundation for quality healthcare and encompasses many measures that patients can pursue and implement in everyday life. In addition to scheduling regular check-ups with primary care healthcare providers, patients can make positive changes in diet and exercise habits, take prophylactic medications, such as aspirin for cardiovascular disease prevention, seek out screening for cancer and sexually transmitted infections (STI), stay up-to-date with immunization schedules, and inform providers of any psychosocial issues, such as depression, substance use issues, and intimate partner violence. A diverse patient population may also have diverse

Corresponding author: Fatma Levent Contact Information: Fatma.levent@ttuhsc.edu DOI: $10.12746 /$ swrccc.v6i25.486 barriers to care, so patient education can be a key factor involved in maintaining healthy behaviors.

Symptom management and preventive measures are increasingly becoming the responsibility of patients with chronic diseases, such as heart disease, stroke, cancer, diabetes, arthritis, respiratory diseases, and oral conditions. ${ }^{1}$ Seven of ten deaths in America are due to chronic diseases, although the overwhelming majority of these conditions are preventable or manageable with lifestyle changes, including physical activity, nutrition, and smoking and alcohol cessation. ${ }^{1}$ Health fairs are one way to improve patient education and patient health self-efficacy, or the extent to which a patient believes he or she can engage in health-promoting behavior. Self-efficacy is highly correlated with compliance and treatment adherence; therefore, increased self-efficacy can improve patient outcomes and health overall. ${ }^{2}$ In fact, self-efficacy is one of the most important predictors of behavior change and maintenance via improved interest, commitment, effectiveness, and 
effort. ${ }^{2,3}$ Improvement of health self-efficacy, patient autonomy and empowerment, and patient education can encourage and maintain lifestyle changes that decrease incidence and morbidity/mortality rates of preventable chronic diseases.

In addition to improving self-efficacy, health fairs can increase access to screening and health information in populations that have low socioeconomic statuses, are uninsured, and are in medically underserved areas. ${ }^{4}$ Because of financial, insurance, cultural, and/or linguistic barriers to care, these populations have limited access to preventive services and therefore experience higher incidences and morbidity/mortality rates of chronic conditions like cancer, heart disease, chronic infections and diabetes. ${ }^{4}$ By providing free and open access to healthcare services independent of insurance or income, health fairs can decrease the incidence of complications and long-term effects of preventable conditions in underserved and disadvantaged populations. This impact can be magnified if health fairs are held in areas with a shortage of primary care health services that are accessible by public transportation. ${ }^{4}$

In this case, Lubbock County is considered partially medically underserved based on the percentage of elderly individuals over age 65 , poverty rate, infant mortality rate, and the ratio of primary care physicians per 1000 people; surrounding areas-including Lamb, Hockley, Terry, Lynn, Garza, Crosby, and Floyd Counties-are considered wholly medically underserved. ${ }^{5}$ Medically underserved populations in Lubbock are the poor and indigent, those struggling with mental and behavioral health issues, minorities and immigrants, the elderly, teens and young adults, children in poverty and in single-parent households, alcohol and drug users, and obese patients. ${ }^{6}$ Myriad barriers affect these populations' access to care, including the lack of education and knowledge of available resources, lack of transportation, socioeconomic status, and attitudes and culture surrounding health and lifestyle choices. ${ }^{6}$ Therefore, although these resources are available in Lubbock County, they may not be fully utilized or understood, thus decreasing the overall population health and contributing to Lubbock's status as a partially medically underserved area. A shortage of rural health physicians and primary care providers may also contribute to West Texas's medically underserved status. In 2010 , one study demonstrated that only $7.1 \%$ of physicians practiced in large rural areas, $2.6 \%$ in small rural, and $1.3 \%$ in remote rural within the United States. ${ }^{7}$ This pervasive and longstanding problem contributes to the increased disparity of health in rural areas compared to urban areas.

Lubbock County and its surrounding areas-including West Texas, Eastern New Mexico, and Southwest Oklahoma-have among the highest percentage of uninsured populations in the United States (Figure 1). ${ }^{8}$ This reflects income and availability of public coverage as well as increased risk among people of color and individuals below the poverty threshold; cost is often the most significant barrier to insurance coverage..$^{9}$ Although the uninsured population percentage has decreased since 2006 (Figure 2), Lubbock County still had an uninsured population rate of $16.9 \%$ in 2015 , with surrounding areas ranging from $13.3 \%$ to $33.3 \%$ and an average of $24.1 \%$ uninsured. ${ }^{8}$ According to the 2016 estimated population census, that means there are approximately 42,675 uninsured individuals in Lubbock County alone. ${ }^{10}$ This substantial population is at risk for developing chronic preventable conditions with ultimately poorer outcomes, and countywide health fairs may provide an opportunity to address these issues.

\section{Methods}

\section{Health Fair Organization}

Fourteen student organizations and two supermarkets collaborated to organize a community health fair in Lubbock, Texas, from 9 a.m. to 5 p.m. The health fair was held at a local supermarket, United Market Street, and was accessible to public transportation. Approximately 50 medical student volunteers provided support on the day of the event. The student organizations involved were the Student National Medical Association (SNMA); American Medical Student Association (AMSA); Cardiology Interest Group (CIG); Dermatology Club, Lubbock Impact Free Clinic, Gay-Straight Alliance 

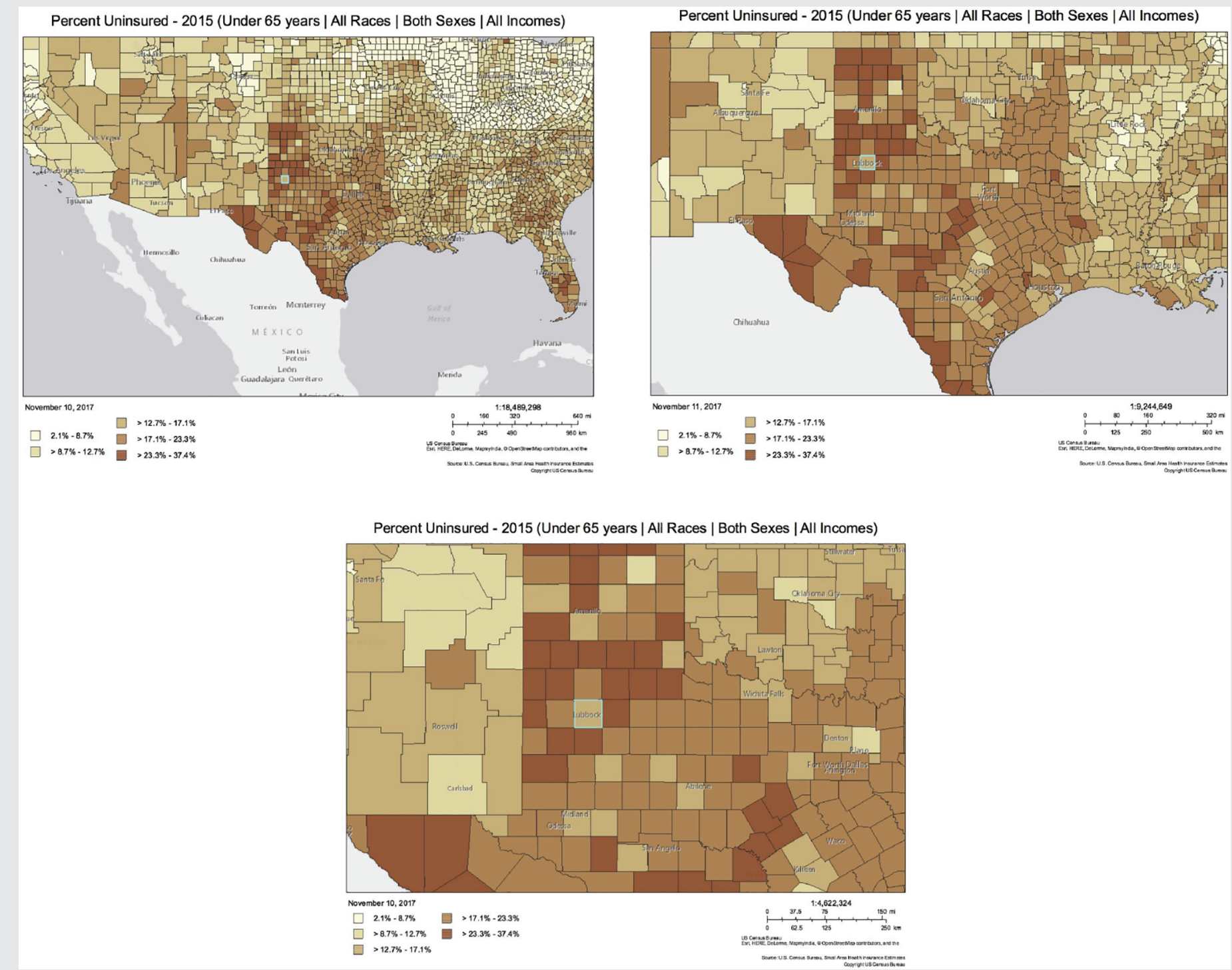

Figure 1. Uninsured population percentages in Southern United States, Texas, and West Texas with Lubbock County highlighted in green. ${ }^{8}$

(GSA); Integrative, Complementary, and Alternative Medicine (ICAM); Internal Medicine Club; Latino Medical Student Association (LMSA); Muslim Health Professions Association; Ob/Gyn Club; Oncology Interest Group; Pediatrics Club; and Psychiatry Club. The supermarkets involved were United Market Street and Natural Grocers. Additionally, the Student National Medical Association donated 27 blood glucose monitors, 17 of which were given to diabetic patients and 10 were donated to the Lubbock Impact Free Clinic.

- AMSA provided services, including body mass index (BMI) calculations and vision and hearing testing.CIG provided blood pressure checks as well as information on heart health, hypertension management, and the Dietary Approaches to Stop Hypertension (DASH) diet. 


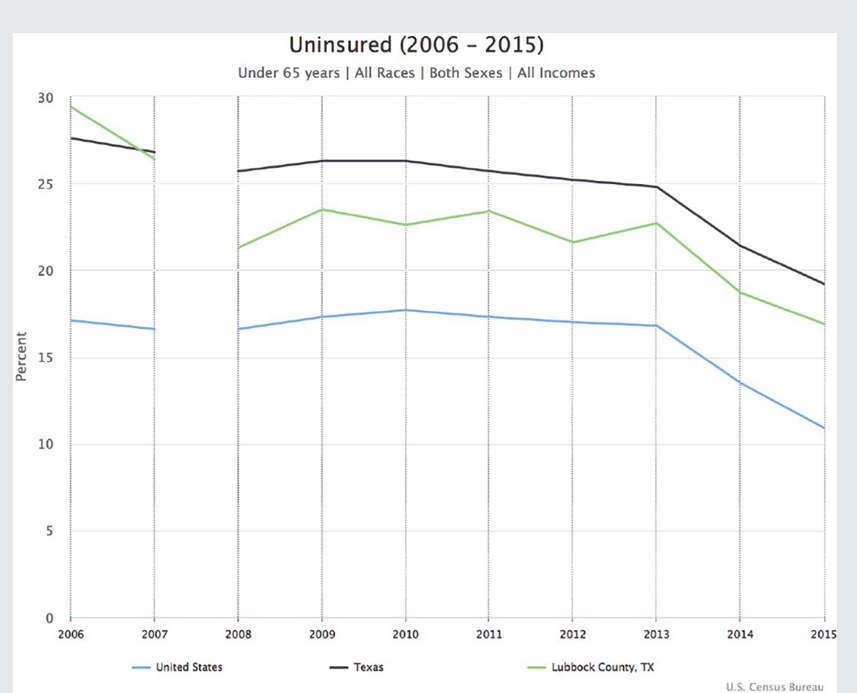

Figure 2. Uninsured populations in United States, Texas, and Lubbock County over time. ${ }^{8}$

- Dermatology Club provided patient education on topics, such as skin care, skin cancer, and screening recommendations.

- Lubbock Impact Free Clinic conducted blood glucose checks and lipid panels.

- GSA provided patient education on LGBTQ (Lesbian, Gay, Bisexual, Transgender, Questioning, Queer) friendly healthcare providers in Lubbock and topics people in the LGBTQ population should talk to their healthcare providers about.

- ICAM provided information on how to read nutrition labels and yoga sequences to reduce stress.

- Internal Medicine Club provided information on vaccinations.

- LMSA provided Spanish-language translations for Spanish-speaking community members.

- Muslim Health Professions Association provided information on stress management and diabetes management.

- Ob/Gyn Club provided patient education on the importance of screenings and recommendations.
- Oncology Interest Group provided information on cancer screenings and recommendations.

- Pediatrics Club provided health-related kid-friendly games and information on nutrition, physical fitness, and hand washing.

- Psychiatry Club provided information on mental health awareness and wellness resources.

- United Market Street recruited its team of registered dietitians to teach guests how to shop for different lifestyles, including heart healthy and gluten-free diets.

- Natural Grocers provided information on nutrition and cooking classes.

\section{HEALTH FAIR PARTICIPANTS}

Students recruited participants by distributing 400 flyers at United Market Street, the Texas Tech University Health Sciences Center and undergraduate campus, the I-27 urgent care clinic, and the student-run free clinic in Lubbock, Texas. The event was also advertised on 3 TV news channels and 1 Spanish-speaking radio station.

\section{MEASURES}

Fifty-seven community members who visited the health fair filled out an evaluation that assessed demographics, which organizations' tables they visited, self-reported self-efficacy, which tables they enjoyed best and why, and comments for potential improvement. Participants rated self-efficacy on a scale from 0 to 10 when asked the question, "Before/ after visiting the community health fair, how confident overall were/are you in your ability to take good care of your health?"

\section{Participant Demographics}

The majority of participants were in their twenties (Figure 3), female (Figure 4), white (Figure 5), and had finished some college (Figure 6). 


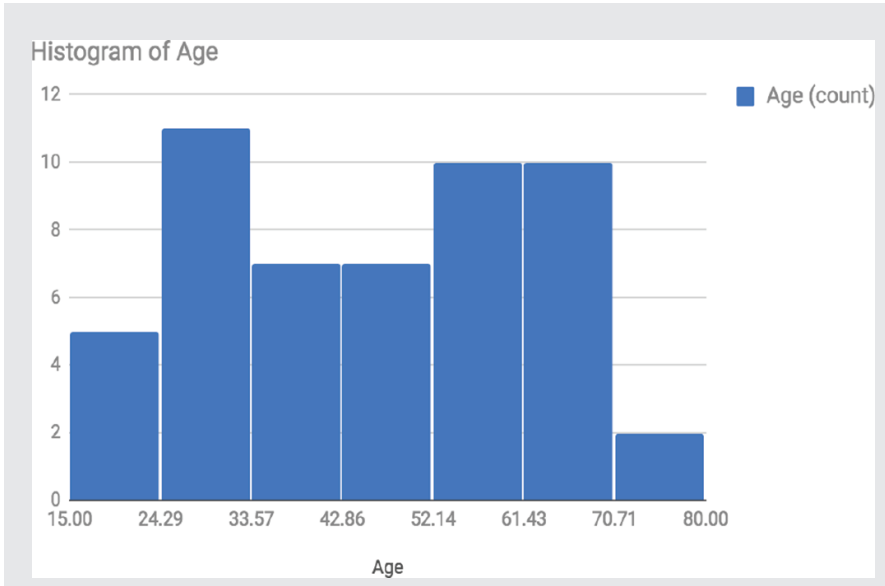

Figure 3. Age of health fair participants.

\section{DATA ANALYSIS}

Data were analyzed using RStudio and Microsoft Excel. Data analyses include descriptive statistics to examine participant demographics, participant self-reported health self-efficacy, and other study variables of interest.

\section{RESULTS}

A one-tailed two-sample t-test revealed that participants' self-reported health self-efficacy improved

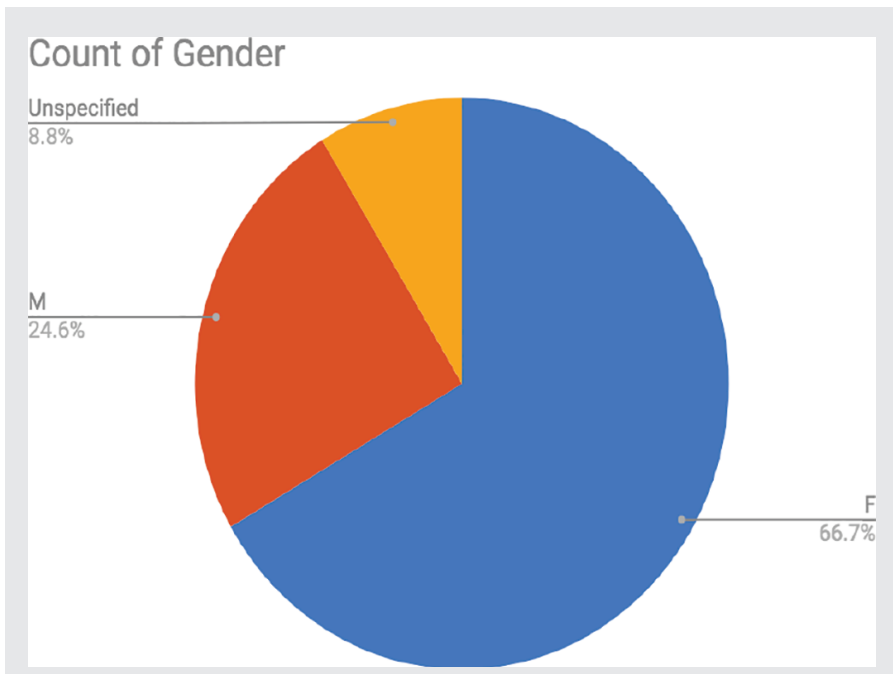

Figure 4. Gender of health fair participants.

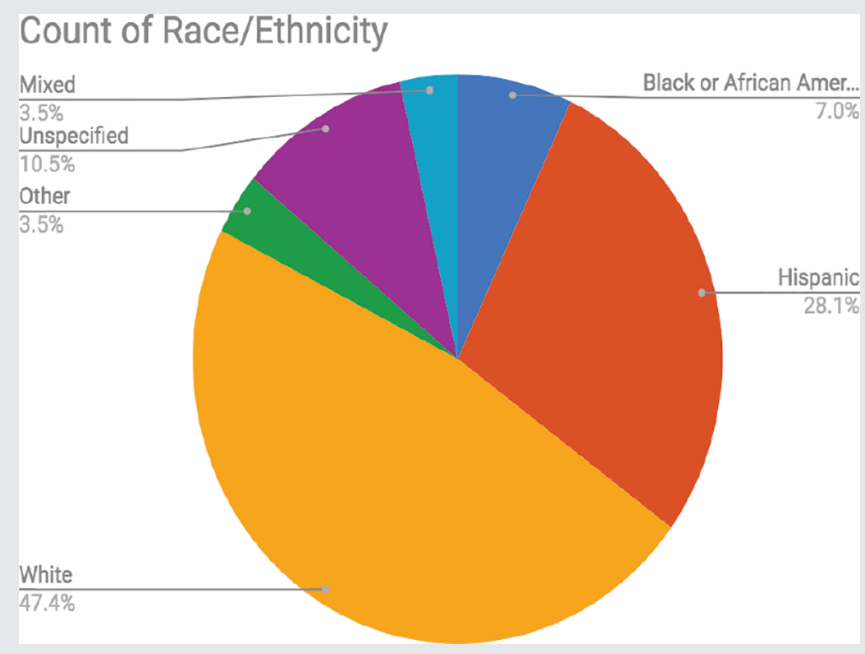

Figure 5. Race/ethnicity of health fair participants.

by 0.87 (before: 7.96 , after: $8.83, p<0.0001$, std $=1.78$ ) on a scale from 0 to 10 after visiting the health fair.

We refrained from presenting analyses that statistically compare subgroups to each other (e.g., age groups, genders, and education levels) because those results are highly driven by chance in this relatively small population especially when not all participants answered every survey question.

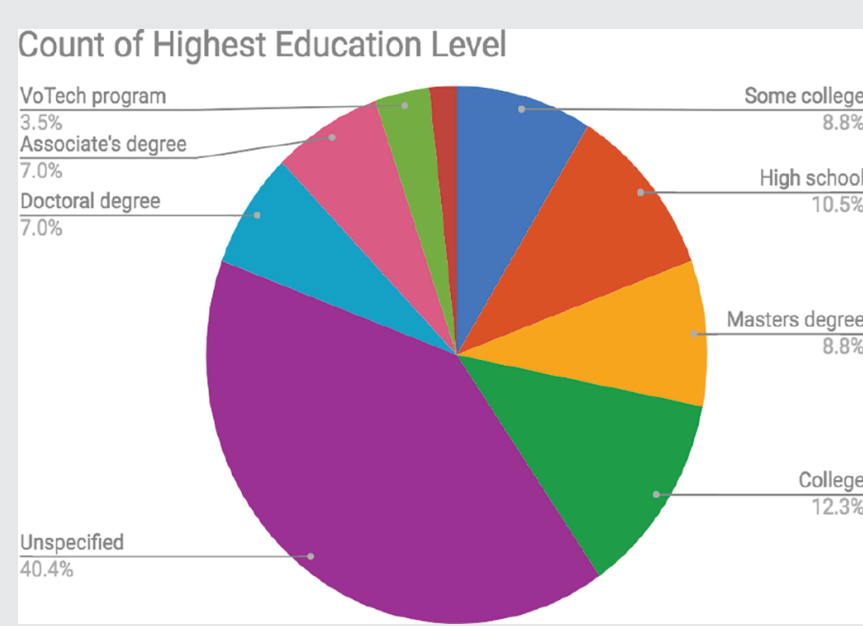

Figure 6. Highest level of education completed by health fair participants. 
The most popular tables, according to participants, were the Pediatrics Club, Free Clinic, and Cardiology Interest Group tables, which were listed as the favorite table of 13,12 , and 11 participants, respectively. This may be a result of the pediatrics station being very interactive and included several activities to participate in. The Free Clinic and Cardiology tables screened for hypertension and diabetes with blood glucose checks, possibly making them more popular.

Each table was visited by the majority of health fair participants, with the most participants visiting the CIG table $(92.98 \%)$ and the fewest participants visiting the GSA table (52.63\%) (Table 1).

Of the 53 participants who had their blood pressure taken, $49.06 \%$ of participants qualified for stage I hypertension with a systolic blood pressure between

Table 1. Percentage of participants who visited each organization's table

\begin{tabular}{|l|c|}
\hline Organization & Percentage of Participants \\
\hline $\begin{array}{l}\text { American Medical Student } \\
\text { Association (AMSA) }\end{array}$ & $78.95 \%$ \\
\hline $\begin{array}{l}\text { Cardiology Interest Group } \\
\text { (CIG) }\end{array}$ & $92.98 \%$ \\
\hline Dermatology Club & $85.96 \%$ \\
\hline Free Clinic & $84.21 \%$ \\
\hline Gay-Straight Alliance (GSA) & $52.63 \%$ \\
\hline $\begin{array}{l}\text { Integrated, Complementary, } \\
\text { Alternative Medicine (ICAM) }\end{array}$ & $73.68 \%$ \\
\hline Internal Medicine (IM) Club & $64.91 \%$ \\
\hline $\begin{array}{l}\text { Muslim Health Professions } \\
\text { Association (MHPA) }\end{array}$ & $75.44 \%$ \\
\hline Ob/Gyn & $68.42 \%$ \\
\hline $\begin{array}{l}\text { Oncology Interest Group } \\
\text { (OIG) }\end{array}$ & $59.65 \%$ \\
\hline Pediatrics Club & $66.67 \%$ \\
\hline Psychiatry Club & $75.44 \%$ \\
\hline Natural Grocers & $75.44 \%$ \\
\hline
\end{tabular}

130 and $139 \mathrm{mmHg}$ and/or diastolic blood pressure between 80 and 89 mmHg. ${ }^{11}$ Furthermore, 35.85\% qualified for stage II hypertension with a systolic blood pressure $\geq 140 \mathrm{mmHg}$ and/or a diastolic blood pressure $\geq 90 \mathrm{mmHg}$. Therefore, only $15.09 \%$ did not fall under hypertensive criteria. Additionally, $75 \%$ had a BMI $\geq 25$ (overweight), and, of these, $41.7 \%$ had a $\mathrm{BMI} \geq 30$ (obese). ${ }^{12}$ Of the eight participants who had a fasting blood glucose test, 3 were normal with a fasting blood glucose level of $\leq 99 \mathrm{mg} / \mathrm{dL}$, 3 had prediabetes with a fasting blood glucose level from 100$125 \mathrm{mg} / \mathrm{dL}$, and 2 had diabetes with a fasting blood glucose level $\geq 126 \mathrm{mg} / \mathrm{dL}$. Of the 55 participants who had a random blood glucose test, 3 had diabetes with a random blood glucose level $\geq 200 \mathrm{mg} / \mathrm{dL}$. The maximum blood glucose level was $442 \mathrm{mg} / \mathrm{dL}$. These cardiovascular risk factors were shared with each at-risk participant, and they were encouraged to follow up with a primary care provider. This may not be representative of the population at large as individuals with hypertension and diabetes mellitus may be more likely to participate in screening.

\section{Discussion}

Although with small magnitude, these results suggest that the community health fair in Lubbock, Texas had a positive impact on community members' health self-efficacy as indicated by improved overall confidence in participants' ability to take good care of their health.

The most popular tables were Pediatrics Club, Free Clinic, and Cardiology Interest Group tables. These findings in addition to comments by participants indicate the need for patient education related to children's health, diabetes and blood glucose management, and diet and physical fitness to maintain a healthy BMI. They also emphasize the importance of the health fair in relation to preventive care services for participants.

The most visited table was the CIG table, and the least visited table was the GSA table. This suggests awareness for the importance of blood pressure checks and hypertension management, and it potentially identifies a medically underserved LGBTQ 
population in Lubbock, Texas. This minority population may not have been reached by the advertisement techniques utilized, which may be addressed in future health fairs to provide a broader outreach.

Upon screening, the majority of participants had risk factors for cardiovascular disease and poor health including hypertension, diabetes, and obesity. Obese patients have been identified as one of the medically underserved populations in Lubbock, Texas; these risk factors in addition to barriers to care and insufficient patient education contribute to the higher incidences and morbidity/mortality rates of chronic conditions. Follow-up with primary care providers and lifestyle changes are essential to improving overall health and controlling risk factors.

Strengths of this study include the wide variety of healthcare services offered by medical students and the fact that it provided a learning opportunity for medical students to practice providing patient education, counseling, obtaining basic vital signs, and checking blood glucose. It also helped identify and help at-risk populations who may have low access or barriers to primary and preventive health care, therefore increasing the accessibility of healthcare services in a rural and underserved area.

Limitations of this study include the fact that the participants reported health self-efficacy on a subjective scale as well as the number of unanswered evaluation questions. Areas for improvement in future health fairs may include recruiting more healthcare groups outside of TTUHSC; expanding community awareness and advertising especially in Spanishspeaking populations; specifically targeting homeless, uninsured, or minority populations; and being more vigilant in ensuring all participants answer all evaluation questions. Future research may include more concrete data that include a larger sample population, before-and-after quizzes testing participants' knowledge on preventive healthcare, comparisons of health self-efficacy between subgroups (e.g., age groups, genders, and education levels) asking visitors to list when they last had a preventive health check-up, and identifying participants' barriers to receiving or engaging in routine health services. Input from participants about best times and locations for health care services and events like this can increase access to interested populations. Furthermore, a future health fair might attempt to track participants who exhibited risk factors for cardiovascular disease to determine whether they followed recommendations to visit a primary care provider. Future health fairs could also evaluate student perceptions of the effects and can analyze what they learned from the health fair.

\section{Conclusions}

While this health fair showed improved self-efficacy among patients, it did not imply long-term health improvements because it did not provide ongoing healthcare services for patients, which is vitally important to maintenance of healthy behaviors. Long-term change in behavior can be hard to accomplish in adult patients, therefore continuous and consistent patient education and awareness about chronic and acute health conditions may be necessary to make profound changes in Lubbock's healthcare on a population level.

Some skepticism exists about the long-term benefits of health fairs and community-based approaches to changing health behaviors. This is in part due to inefficient study designs, costly initiatives, and short-term patient education efforts. ${ }^{13}$ Community-wide health promotion efforts in the past have suggested a modest impact at best because an individual's behavior is shaped by dynamic influences in interpersonal, organizational, community, and policy environments. ${ }^{14}$ Thus, continuous effort and change on a social level must be pursued to make and sustain lasting change in patients' healthy behaviors. Even self-efficacy itself is highly dependent on social support and environmental influences. ${ }^{2}$ This does not necessarily mean that community-wide health fairs like the one discussed in this article should be discontinued. Rather, it suggests that study designs should be more efficient and cost effective to better gauge community involvement and impact; patients should have individually tailored treatment by a provider who fully understands their social, financial, and cultural environment; and long-term goals 
and challenges for both the patient and provider should be addressed by encouraging continuous and consistent visits with a preventive and primary care provider.

Acknowledgements: We would like to thank Texas Tech University Health Sciences Center, School of Medicine, the Office of Student Services, the Student National Medical Association, and all student organizations that made this event possible. Student organizations included American Medical Student Association (AMSA); Cardiology Interest Group (CIG); Dermatology Club; Lubbock Impact Free Clinic; GayStraight Alliance (GSA), Integrative, Complementary, and Alternative Medicine (ICAM); Internal Medicine Club; Latino Medical Student Association (LMSA); Muslim Health Professions Association; Ob/Gyn Club; Oncology Interest Group; Pediatrics Club; and Psychiatry Club.

Article citation: Lindgren $\mathrm{TH}$, Burroughs $\mathrm{C}$, Burroughs M, Parker G, Levent F. Improving health self-efficacy at a community health fair in Lubbock, Texas. The Southwest Respiratory and Critical Care Chronicles 2018; 6 (25):52-59

From: School of Medicine (THL, CB, MB, GP) and Department of Pediatrics (FL) at Texas Tech University Health Sciences Center, Lubbock, Texas

Submitted: $4 / 5 / 2018$

Accepted: 6/15/208

Reviewer: Patti Patterson MD

Conflicts of interest: none

This work is licensed under a Creative Commons Attribution-ShareAlike 4.0 International License

\section{REFERENCES}

1. Centers for Disease Control and Prevention. Chronic diseases: The power to prevent, the call to control. National Center for Chronic Disease Prevention and Health Promotion, 2009.

2. Martos-Méndez MJ. Self-efficacy and adherence to treatment: The mediating effects of social support. J Behavior, Health, Social Issues 2015 Nov; 7(2):19-29.

3. Bandura A. Perceived self-efficacy in cognitive development and functioning. Educational Psychologist 1993;28(2): $117-148$.

4. Murray K, Liang A, Barnack-Tavlaris J, et al. The reach and rationale for community health fairs. J Cancer Education 2014 Mar; 29(1):19-24.

5. Cancer Prevention Research Institute of Texas. Medically Underserved Areas: Texas Department of Rural Affairs. Texas Department of Rural Affairs, 2009.

6. University Medical Center. Community Health Needs Assessment. BKD, LLP, 2013.

7. Agency for Healthcare Research and Quality. Primary Care Workforce Facts and Stats No. 3, 2010.

8. U.S. Census Bureau. Small Area Health Insurance Estimates (SAHIE), 2015.

9. Kaiser Family Foundation. Key Facts about the Uninsured Population. 2017.

10. U.S. Census Bureau. 2016 Population Estimates for Lubbock County, Texas. 2016.

11. Journal of the American College of Cardiology. 2017 Guideline for the Prevention, Detection, Evaluation and Management of High Blood Pressure in Adults. 2017.

12. World Health Organization. BMI Classification. 2017.

13. Thompson B, Coronado G, Snipes SA, et al. Methodologic advances and ongoing challenges in designing communitybased health promotion programs. Ann Rev Public Health 2003 May; 24(1):315-340.

14. Merzel C, D'Afflitti J. Reconsidering community-based health promotion: promise, performance, and potential. Am J Public Health 2003 Apr; 93(4):557-74. 